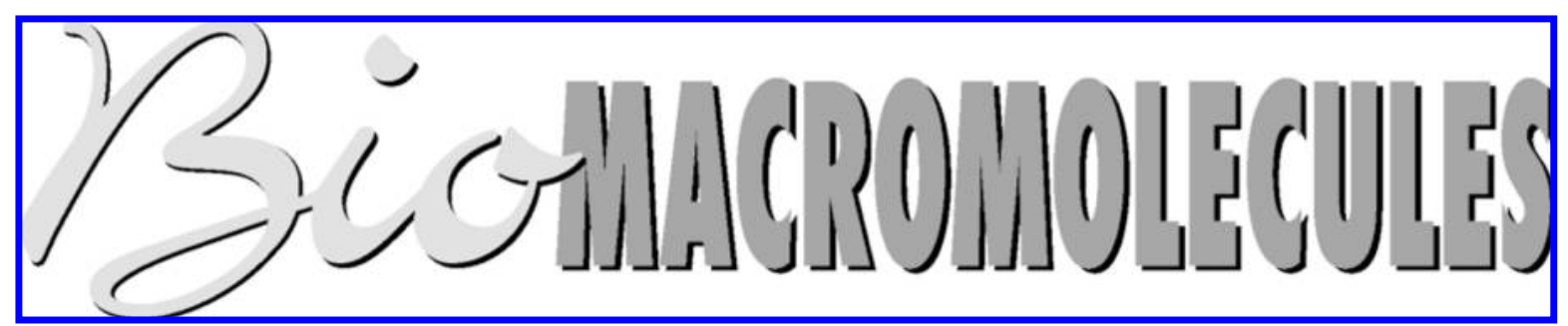

March 2009

\title{
Communications
}

\section{Innovative Approach for Producing Injectable, Biodegradable Materials Using Chitooligosaccharides and Green Chemistry}

\author{
Luciano F. Boesel, ${ }^{\dagger}$ Rui L. Reis, ${ }^{\ddagger, \S}$ and Julio San Román ${ }^{\star, \S}$ \\ Department of Biomaterials, Institute of Science and Technology of Polymers, Spanish National Research \\ Council (CSIC), 28006, Madrid, Spain, 3B's Research Group-Biomaterials, Biodegradables and \\ Biomimetics, Department of Polymer Engineering, University of Minho, Campus of Gualtar, \\ 4710-057, Braga, Portugal, and IBB-Institute for Biotechnology and Bioengineering, PT Government \\ Associated Laboratory, Braga, Portugal
}

Received November 18, 2008; Revised Manuscript Received January 19, 2009

\begin{abstract}
Although there are a number of injectable biomaterials currently under development, they present some drawbacks such as being based on synthetic polymers, needing toxic or aggressive synthesis procedures or using raw materials with low availability and/or high production costs. Having this in mind, a novel injectable biomaterial using chitooligosaccharides as starting materials was developed. This system uses a widely available and cheap polymer from marine biomass (chitosan), which can be turned into an injectable material by water-based and ecologically friendly reactions. Chitooligosaccharides were functionalized with methacrylic groups, to allow in situ crosslinking. The degree of substitution, as determined by ${ }^{1} \mathrm{H}$ NMR, varied between 5 and $50 \%$. The system was characterized in terms of kinetics of gel formation, rheology, degradation behavior and in vitro cytotoxicity. The gelation time could be easily tailored between 1.5 and $60 \mathrm{~min}$ by changing the conditions of the methacrylation reaction, and the final gel presented rheological properties typical of strong gels, that is, shear stresses in the $\mathrm{kPa}$ range. The cross-linked gel was degradable and nontoxic, presenting indeed an interesting cytokinetic effect. Injectable materials based on chitooligosaccharides are, therefore, an innovative system combining adequate biological performance, ease of preparation, and an ecologically friendly concept of production.
\end{abstract}

\section{Introduction}

Injectable systems are an important class of materials employed in the biomedical field. Besides the long-term use of acrylic polymers or calcium-phosphate ceramics as bone cements, recently injectable materials made of biodegradable polymers have drawn considerable attention. The potential applications of injectable biodegradable systems (IBS) are mainly as tissue engineering scaffolds or controlled drug delivery systems. Injectable materials eliminate the need of complicated surgery for implantation and are able to take the exact shape of the defect to be regenerated, thus eliminating the need for precise prefabrication of the scaffold. Moreover, they may be easily

* To whom correspondence should be addressed. E-mail: jsroman@ ictp.csic.es.

CSIC.

University of Minho.

$\S$ IBB. mixed with cells, growth factors, or drugs just before implantation, making the handling of such agents much easier. Depending on the composition and hardening mechanism of the injectable material, both hard and soft tissues may be targeted. In general, in situ cross-linking systems lead to stiffer and stronger materials; thermoresponsive hydrogels or self-assembly systems, on the other hand, are weaker, although they raise less cytotoxicity issues due to the lack of solvents or initiators. ${ }^{1,2}$

In recent years, the attention has moved from the traditional, nondegradable injectable materials such as poly(isopropylacrylamide) (PNIPA) or Pluronics to the more appealing degradable counterparts. $^{3-5}$ The latter includes di-, tri-, or multiblock copolymers of poly(lactic-co-glycolic acid) (PLGA) and poly(ethylene glycol) (PEG) ${ }^{6,7}$ and cross-linkable systems of acrylated poly(propylene fumarate) (PPF) ${ }^{8,9}$ or poly(anhydrides), ${ }^{10,11}$ among other synthetic polymers. Although these and other polymers have fulfilled all the requirements for being used as 
injectable materials in tissue engineering and drug delivery applications, their synthesis is time-consuming and costly and makes use of toxic solvents or substances, with a consequent need for purification steps. To counteract that, natural polymers, specially chitosan, alginate and gelatin, have also been employed in the preparation of injectable biomaterials. ${ }^{4,5}$ In most cases, however, the thermogelling effect is only obtained when high amounts of a synthetic polymer such as PNIPA or Pluronics are grafted on the biopolymer backbone. ${ }^{12-14}$ Such methodology eliminates the biobased appeal of these systems once they are more like natural-"containing" than natural-"based" polymers.

In this context, an innovative natural-based IBS was developed. This system is mainly constituted by biobased materials, and a "green" chemistry approach is used throughout the fabrication process. To that, chitooligosaccharides ("oligomers" of chitosan) were used as the precursor, and a simple methacrylation reaction was employed to incorporate polymerizable double bonds to the oligomers. In this communication the synthesis and in vitro characterization of such systems are reported.

\section{Experimental Section}

Materials and Reagents. Chitosan from crab shells (85\% deacetylated, $\mathrm{Mv}=640 \mathrm{kDa}, 97 \%$ pure) was obtained from Idebio S. L., Spain. The pectinase for depolymerization was Multifect Pectinase FE, from Genencor International B. V. (The Netherlands), with an activity of $180 \mathrm{U} / \mathrm{g}$. All other reagents and solvents (acetic acid, methacrylic anhydride, sodium persulfate, ascorbic acid) were reagent grade and used as received. Phosphate buffered saline (PBS) was prepared by dissolving one pouch (Sigma-Aldrich) in $1 \mathrm{~L}$ of distilled water.

Preparation of Methacrylated Chitooligosaccharides. Chitosan was first purified by dissolution in diluted acetic acid and reprecipitation in aqueous sodium hydroxide. It was then washed several times and freeze-dried for further use.

Depolymerization was performed in acetate buffer (acetic acid/ sodium hydroxide, $\mathrm{pH}$ 5.5). After preliminary tests to define the optimum conditions of temperature, time, agitation, and ratio of enzyme, these were defined as $50{ }^{\circ} \mathrm{C}, 17 \mathrm{~h}, 60 \mathrm{rpm}$, and $1 \mathrm{~g}$ of enzyme per $100 \mathrm{~g}$ of chitosan, respectively. After the depolymerization step, the solution was heated at $100{ }^{\circ} \mathrm{C}$ for $10 \mathrm{~min}$ and filtered (to denature and remove the enzyme), precipitated in $90 \%$ ethanol and centrifuged (to remove low molecular weight oligomers). The oligomers were then redissolved in water, washed in $90 \%$ ethanol, and centrifuged three more times (to ensure complete removal of acid and sodium hydroxide) before being vacuum-dried.

Methacrylation of chitooligosaccharides was performed in PBS under different conditions: (a) time, from 15 to $45 \mathrm{~min}$; (b) temperature, from 25 to $50{ }^{\circ} \mathrm{C}$; (c) concentration of oligomer in PBS, from 2.5 to $7.5 \%$; (d) molar ratio of methacrylic anhydride per $\mathrm{NH}_{2}$ groups in chitosan, from 0.125 to 0.268 . The different conditions yielded different degrees of substitution on chitooligosaccharides (see Results and Discussion). After preliminary tests and optimization using factorial planning, the standard conditions used to obtain samples for characterization studies were $50{ }^{\circ} \mathrm{C}, 15 \mathrm{~min}, 2.5 \%$ of oligomer in PBS (w/v), and molar ratio of 0.268 .

Analysis of Modified Chitooligosaccharides. Matrix-assisted laser desorption ionization/time-of-flight mass spectrometry (MALDI-TOF/ MS) was performed on a Voyager-DE PRO equipment (Applied Biosystems) using 2,5-dihydroxybenzoic acid as the matrix. The oligomer was dissolved $(1 \% \mathrm{wt} / \mathrm{vol})$ in $0.175 \mathrm{M}$ acetic acid. Mass spectra were recorded in the positive ion mode using a nitrogen laser $(337 \mathrm{~nm})$. All spectra were measured in the linear mode.

Proton nuclear magnetic resonance $\left({ }^{1} \mathrm{H}\right.$ NMR) was performed by dissolving oligomers in deuterium chloride (35 wt \%, Aldrich)/ deuterium oxide $(99.9 \%$, Merck). Spectra were acquired with a Inova
$400 \mathrm{MHz}$ equipment (Varian), at $60^{\circ} \mathrm{C}$, with a relaxation delay of $6 \mathrm{~s}$ and $45^{\circ}$ pulses. The degree of deacetylation was calculated according to ref 15

$$
\operatorname{DDA}(\%)=\left(\frac{\mathrm{H} 1(\mathrm{D})}{\mathrm{H} 1(\mathrm{D})+\mathrm{CH}_{3}(\mathrm{~A}) / 3}\right) \times 100
$$

Analogously, degrees of substitution were calculated as

$$
\mathrm{DS}(\%)=\left(\frac{\mathrm{CH}_{3}(\mathrm{M}) / 3}{\mathrm{H} 1(\mathrm{D})+\mathrm{CH}_{3}(\mathrm{~A}) / 3+\mathrm{CH}_{3}(\mathrm{M}) / 3}\right) \times 100
$$

where H1(D) corresponds to the integral of the peak of the first proton of the glucosamine unit, $\mathrm{CH}_{3}(\mathrm{~A})$ to the peak of the methyl group of $\mathrm{N}$-acetyl glucosamine, and $\mathrm{CH}_{3}(\mathrm{M})$ to the peak of the methyl group of $\mathrm{N}$-methacrylamide glucosamine. Standard methacrylation conditions described in the previous section yielded oligomers with DS of $19 \%$, and these were the samples used for characterization studies.

Analysis of the Polymerization Process. For gelation time and temperature for the tube inversion method (TIM), methacrylated chitooligosaccharides $(10 \% \mathrm{wt} / \mathrm{vol})$ and ascorbic acid $(0.025 \mathrm{M})$ were dissolved in PBS and equilibrated at $37{ }^{\circ} \mathrm{C}$; then a solution of $20 \%$ (wt/vol) of sodium persulfate in PBS was added to yield a final concentration of $0.062 \mathrm{M}$ in the oligomer solution. Each $30 \mathrm{~s}$, the tube was removed from the water bath and inverted; the time at which no flow was observed was considered as the gelling time. For determining the maximum temperature of polymerization, a thermocouple with digital display was immersed in the solution at $37^{\circ} \mathrm{C}$ before the addition of the persulfate. Temperature was acquired each $20 \mathrm{~s}$ for $15 \mathrm{~min}$.

For polymerization studies in NMR, the $\mathrm{pH}$ of the solution was adjusted with sodium deuteroxide $\left(40 \%\right.$ in $\mathrm{D}_{2} \mathrm{O}$, Alfa Aesar), and temperature of acquisition was changed to $37^{\circ} \mathrm{C}$. A first spectra was recorded without the initiator redox pair to equilibrate the sample and adjust equipment parameters; then the redox pair was added and mixed, and the tube was immediately inserted in the magnet to record the double bond conversion.

For oscillatory rheometry, the oligomer solution containing AA $(0.025 \mathrm{M})$ in PBS was inserted between the parallel plates $(40 \mathrm{~mm})$ of an Advanced Rheometer 1000 (TA Instruments), and the temperature was adjusted to $37^{\circ} \mathrm{C}$. Next, the solution of NaPS was added and time sweep measurements were started with the following conditions: frequency of $1 \mathrm{rad} / \mathrm{s}$, oscillation stress of $0.4 \mathrm{~Pa}$, and plates gap of 1 $\mathrm{mm}$. For frequency sweep tests, frequency was varied between 0.75 $\mathrm{Hz}$ and $2.50 \mathrm{~Hz}$ and oscillation strain was fixed at 0.10 (up to 17.5 $\mathrm{min}$ ) and 0.01 (afterward) to ensure linear viscoelasticity throughout the experiment. Gel time was defined as the time when $\tan \delta$ was independent of the frequency when plotted against polymerization time.

In Vitro Evaluation of Cytotoxicity and Degradation. Degradation tests were performed by immersing cylindrical specimens of injectable materials in PBS supplemented with $2 \mathrm{U} / \mathrm{mL}$ of lysozyme immediately after polymerization. A ratio of $2.78 \mathrm{~mL}$ of PBS per $1 \mathrm{~g}$ of injectable oligomer was employed for all specimens. Chitosan membranes were used as controls under the same conditions. To avoid accounting for unpolymerized oligomers and soluble activators on the degradation studies, both cylinders and membranes were washed for $2 \mathrm{~h}$ in PBS at $37{ }^{\circ} \mathrm{C}$. At several periods up to 4 weeks, aliquots of the solution were removed and fresh solution was added to keep volume and enzyme concentration constant. At 7, 9, and 11 weeks, the whole solution was changed. Degradation solutions were subjected to high-pressure liquid chromatography (HPLC) analysis in a Shimadzu LC-20AD equipment, using a Phenomenex Luna- $\mathrm{NH}_{2}$ column in reversed mode. Mobile phase was PBS, flow rate was $1 \mathrm{~mL} / \mathrm{min}$, column temperature was $40{ }^{\circ} \mathrm{C}$, and a volume of $100 \mu \mathrm{L}$ was injected. The chromatograms for each specimen were compared with standards of glucosamine, N-acetyl glucosamine, PBS with lysozyme, pristine chitooligosaccharides, and methacrylated oligosaccharides to identify the eluting peaks. The total amount of chitooligosaccharides released to the solution was determined by using the colorimetric method developed by Dubois et al. ${ }^{16}$ Briefly, $0.1 \mathrm{~mL}$ of the solution was mixed with $0.1 \mathrm{~mL}$ of phenol solution $(5 \%$ 


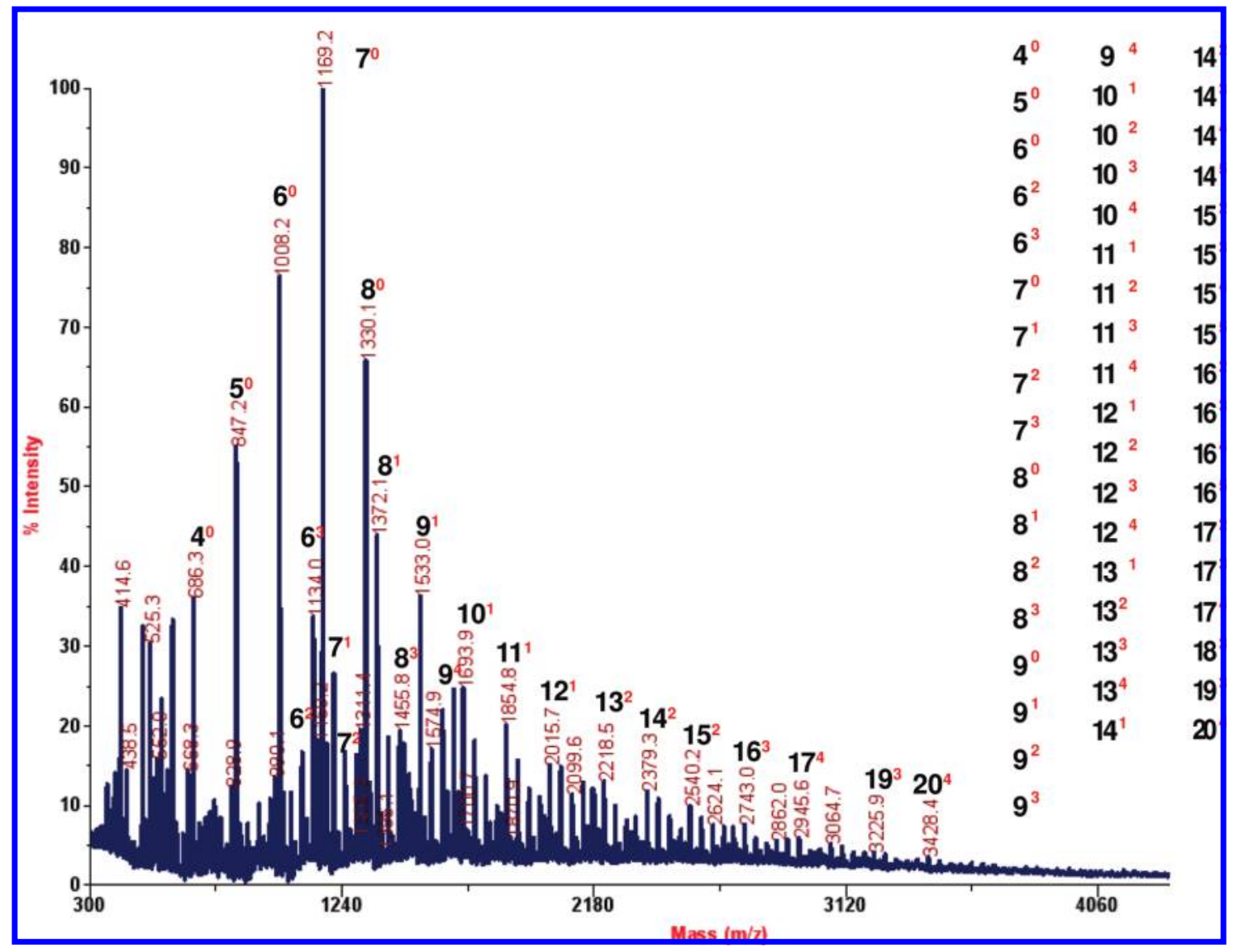

Figure 1. MALDI-TOF/MS spectrum of oligomer mixture after $24 \mathrm{~h}$ of depolymerization. Each peak corresponds to a fragment with (Dp) (Ac) $^{\text {, }}$ where $\mathrm{Dp}$ is the degree of polymerization and $\mathrm{Ac}$ is the number of acetylated units. The columns on the right list all fragments identified for this sample.

in water) and with $0.5 \mathrm{~mL}$ of concentrated sulfuric acid. The mixture was equilibrated at $30{ }^{\circ} \mathrm{C}$ during $20 \mathrm{~min}$ and then the absorbance was read in a Synergy-HT spectrophotometer (Bio Tek Instruments) at 485 $\mathrm{nm}$. A calibration curve was prepared with the pristine chitooligosaccharides.

Cytotoxicity was evaluated with a fibroblast cell line using the MTT assay with the materials' extracts. Injectable specimens were prepared as stated previously, except that the solutions were first sterilized through filtration with a $22 \mu \mathrm{m}$ sterile filter. The specimens were polymerized directly inside falcon tubes, after which cell culture medium was added at a ratio of $0.05 \mathrm{~g}$ of material in $5 \mathrm{~mL}$ of medium. The toxicity of methacrylated oligomers was studied by adding the oligomer solution without activators to the falcon tube. Materials were left at $37{ }^{\circ} \mathrm{C}$ under agitation for 1,2 , and 7 days, being the medium collected and changed by fresh one at each intermediate period. The results were compared with chitosan membranes (whose extracts were obtained in the same manner), negative control (Thermanox) and positive control (Triton $\mathrm{X}-100,0.5 \%$ in culture medium). The assay was then performed as follows: cells were seeded on a 96 well culture plate and incubated until reaching confluence at a density of $5.5 \times 10^{4}$ cells $/ \mathrm{mL}$. The culture medium was then replaced by the eluted extracts of each material $(100 \mu \mathrm{L} / w e l l)$, and the plate was incubated at $37{ }^{\circ} \mathrm{C}$, $5 \% \mathrm{CO}_{2}$, and $24 \mathrm{~h}$. After this period, extracts were removed and ( $(3-$ [4, 5 dimethylthyazol-2-yl])-2,5-diphenyl tetrazolium bromide) (MTT), dissolved in PBS $(0.05 \% \mathrm{wt} / \mathrm{vol})$, was added to each well $(100 \mu \mathrm{L} /$ well). The plate was again incubated at $37^{\circ} \mathrm{C}$ for $4 \mathrm{~h}$, the MTT solution was removed and replaced by $100 \mu \mathrm{L}$ of dimethylsulfoxide (DMSO). The absorbance of the final solution was read in the Synergy-HT spectrophotometer (Bio Tek Instruments) at $570 \mathrm{~nm}$.

\section{Results and Discussion}

Chitooligosaccharides were used to prepare injectable systems, instead of chitosan itself, due to several reasons. First, they are readily soluble in water at any $\mathrm{pH}$, allowing for a higher number of possible chemical modifications as compared to chitosan, which is only soluble in acidic conditions. Second, solutions of oligomers may be prepared at higher concentrations (up to $20 \%$, depending on the degree of polymerization) while still keeping low viscosity. Third, oligomers are easier to modify, and reactions with oligomers proceed faster than reactions with chitosan due to their reduced molecular weight. Chitooligosaccharides were prepared by well-established enzymatic depolymerization reactions. Pectinase, a commercially available enzyme with chitosanase activity and which was shown to depolymerize chitosan into oligomers with medium to high degree of polymerization $(\mathrm{Dp})^{17,18}$ was employed for this purpose. Figure 1 displays a MALDI-TOF mass spectrum of the oligomer mixture after $24 \mathrm{~h}$ of depolymerization. The major components of the mixture are chitooligosaccharides with Dp $5-8$, with none or only one $\mathrm{N}$-acetylated unit. For our purposes, it was important to balance full solubility (what requires the preparation of oligomers with low Dp) with the ability to incorporate at least two modified units in the oligomer backbone (what requires oligomers with high Dp). Therefore, depolymerization time was adjusted to $17 \mathrm{~h}$, a condition leading to a $100 \%$ soluble product and a high fraction of high Dp oligomers. Low Dp chitooligosaccharides were subsequently removed from the mixture by fractional precipitation in ethanol. ${ }^{18}$ 


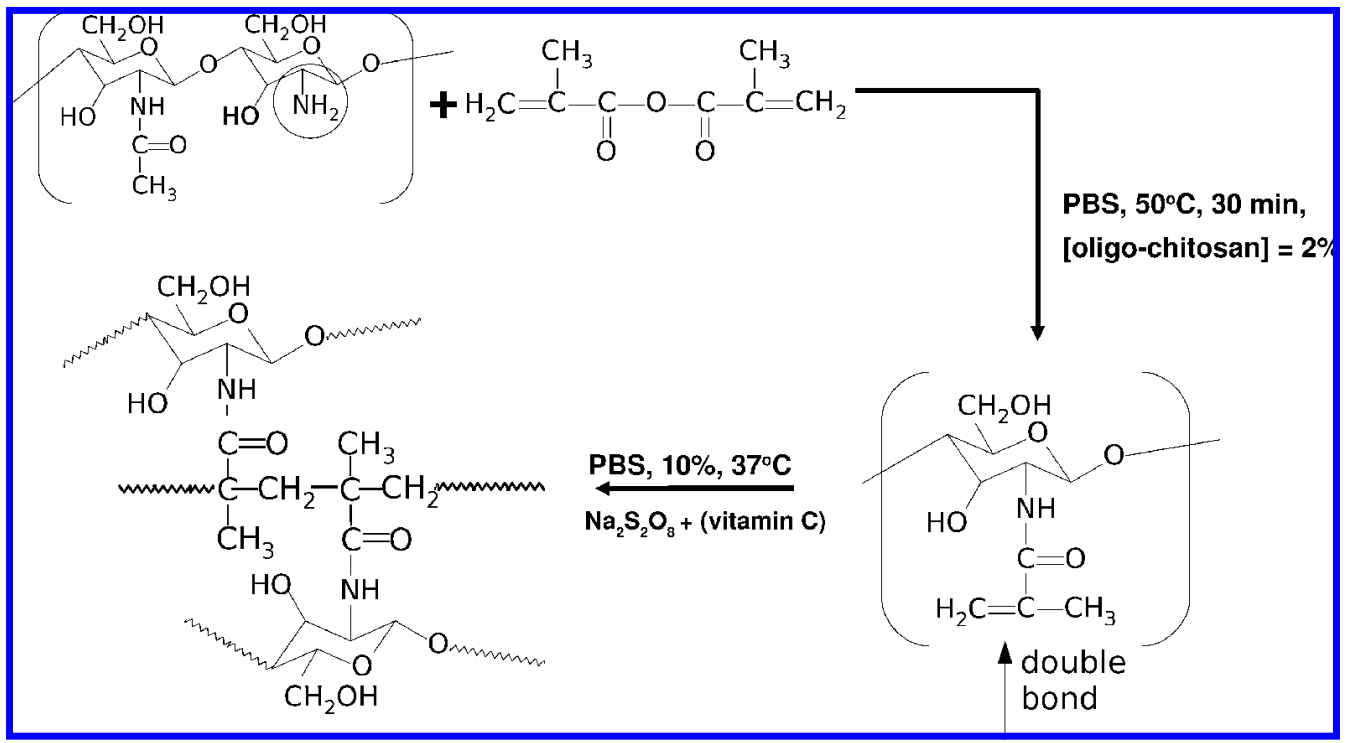

Figure 2. Scheme showing the methacrylation reaction and the cross-linking mechanism initiated by a redox pair of sodium persulfate/ascorbic acid.

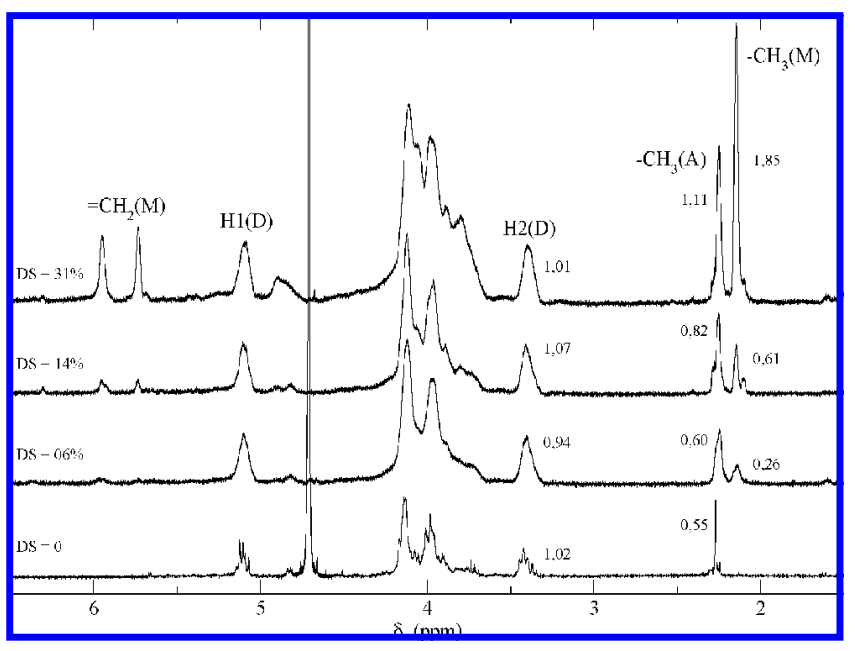

Figure 3. ${ }^{1} \mathrm{H}$ NMR of chitooligosaccharide (bottom) and three modified oligomers with different degrees of substitution (DS). The characteristic bands of each unit in the oligomer backbone are identified: H1(D) and $\mathrm{H} 2(\mathrm{D})$ are the protons at the first and second carbons, respectively, of D-glucosamine; $\mathrm{CH}_{2}(\mathrm{M})$ are the two vinyl protons of the methacrylamide-glucosamine; $\mathrm{CH}_{3}(\mathrm{~A})$ and $\mathrm{CH}_{3}(\mathrm{M})$ are the methyl protons of $\mathrm{N}$-acetyl-glucosamine and methacrylamide-glucosamine, respectively. The integration values for peaks used to determine DA and DS are shown. The peak at $5.1 \mathrm{ppm}(\mathrm{H} 1(\mathrm{D}))$ was given a relative intensity of 1.0.

The remaining oligomer fraction, with Dp higher than 4 (about $80 \%$ of the initial chitosan mass), was then reacted with methacrylic anhydride in PBS (pH 7.4; see Experimental Section). The reaction occurs through nucleophilic substitution at the primary amine, with the release of methacrylic acid to the reaction medium (see scheme in Figure 2). The degree of substitution could be easily tailored by controlling reaction conditions and varied between 5 and $50 \%$. Figure 3 displays the ${ }^{1} \mathrm{H}$ NMR of both native and modified oligomers, with the bands attributed to methacrylamide oligomers appearing at 2.15 ppm ( $\alpha-\mathrm{CH}_{3}$ group) and 5.7-6.0 ppm (methacrylic double bond hydrogens). Although our design of experiments indicated that the temperature has no effect on the degree of substitution (DS), usually a higher temperature was used to allow easier equilibration of the system. The product was soluble in water and was recovered by precipitation in ethanol. Nonetheless, conditions leading to higher degree of substitution were also favoring the appearance of a high fraction of insoluble material. This turned out to be a fraction of oligomers with even higher degree of substitution (as seen by FTIR; data not shown). Overall, DS on the range $16-21 \%$ were the ideal ones, leading to a yield of $80 \%$ relative to the initial oligomer mass (therefore, the final yield of both steps was about $65 \%$ of the initial chitosan mass).

The presence of a polymerizable double bond as a side group on the chitooligosaccharides backbone allowed the cross-linking of these oligomers in physiological conditions, either by a redox initiation or by a photoinitiation system. Irgacure 2959 may be used for the latter, while sodium persulfate (NaPS) and ascorbic acid (AA) form a suitable redox pair initiator. Both systems were shown to be noncytotoxic. ${ }^{19,20}$ In this work, the redox pair at concentrations $0.062 \mathrm{M} / 0.025 \mathrm{M}$ (NaPS/AA) for a solution of PBS containing $10 \%$ (w/v) oligomer was used. The crosslinking occurs through free radical polymerization of the pendant methacrylic groups, as shown schematically in Figure 2. This yielded an injectable system able to gel in $13 \mathrm{~min}$ at $37^{\circ} \mathrm{C}$, as determined by the tube inversion method (TIM). In fact, the system allowed an easy tailoring of gelling time, as it could be changed from $1.5 \mathrm{~min}$ up to $60 \mathrm{~min}$ by changing several factors: concentration of oligomer in PBS, concentration of the redox pair, degree of acetylation (DA) of the native oligomer, and DS of the modified oligomer. The use of methacrylated chitooligosaccharides has an additional advantage over other natural or synthetic polymers, because the persulfate reacts directly with saccharides to produce radicals on the polymer backbone, ${ }^{21}$ avoiding the need of AA (more toxic than the persulfate $)^{19}$ or allowing for the use of lower amounts of the NaPS/AA pair. Systems consisting only of oligomer (10\% w/v) and $\operatorname{NaPS}(0.12 \mathrm{M})$ were able to gel at $37{ }^{\circ} \mathrm{C}$, supporting this hypothesis. Although both the initiation and the propagation steps are exothermic, due to the high water content of the system only a small increase in temperature (to $39.5^{\circ} \mathrm{C}$ ) was observed in the first minute. This increase is most likely due to the decomposition of the redox pair and should therefore present no problem because the temperature quickly returns to the physiological value.

The evolution of viscosity and storage modulus $\left(G^{\prime}\right)$ of the curing gel was followed by oscillatory rheometry using a parallel plates configuration, and is shown in Figure 4. During the initial 


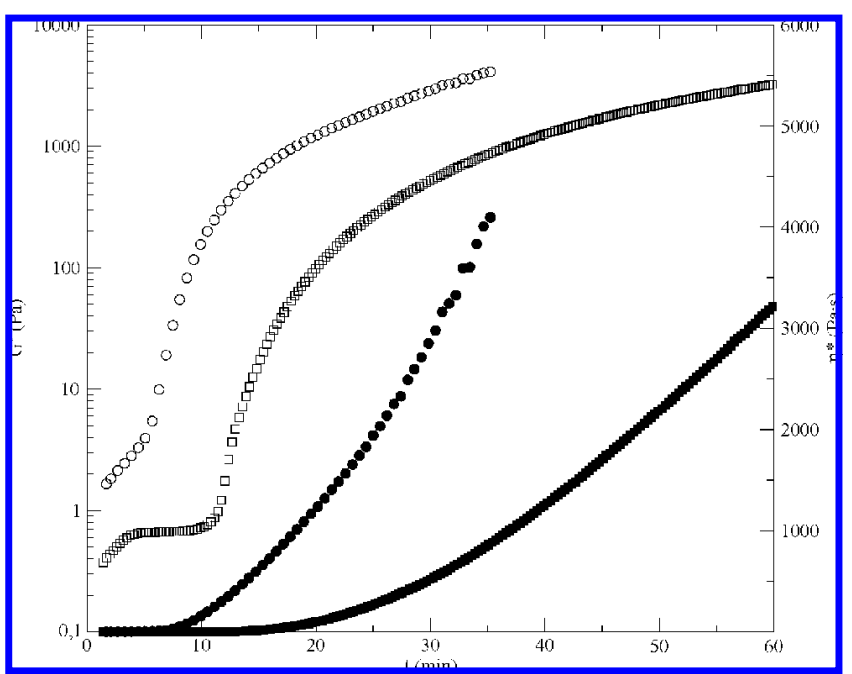

Figure 4. Evolution of storage modulus ( $G^{\prime}$, open symbols) and complex viscosity ( $\eta^{*}$, closed symbols) as a function of polymerization times at two temperatures: $25^{\circ} \mathrm{C}$ (squares) and $37^{\circ} \mathrm{C}$ (circles).

stages of curing, the solution has a very low complex viscosity $\left(\eta^{*}\right)$, close to that of water (lower than $10 \mathrm{~Pa} \cdot \mathrm{s}$ ). This allows easy injection through standard needles or even filtration through filters with pores of $22 \mu \mathrm{m}$ diameter. Close to the gelling time, the system still presents a complex viscosity lower than 500 $\mathrm{Pa} \cdot \mathrm{s}$, facilitating injection. Although the material does not flow anymore after this time, it continues to harden, reaching final values of storage modulus in the range of 4 to $5 \mathrm{kPa}$, which is in the range of strong gels and 1 order of magnitude higher than typical thermogelling or self-assembling systems. ${ }^{7,6,22-24}$ The material is, therefore, easily injectable, fast gelling, and strong and cohesive after final hardening.

The curing process was also studied from a molecular point of view by using oscillatory rheometry. When a cross-linking polymer is at the gel time (time at which it reaches infinite molecular weight), the " $\tan \delta$ " value, as determined from oscillatory rheometry, is independent of the frequency of oscillation. ${ }^{25}$ By rapidly changing the frequency when testing our materials, we obtained a gel time of $15 \mathrm{~min}$, which is quite close to the time of no-flow determined by TIM. In fact, it seems that flow is restricted even before a fully cross-linked polymer is obtained. Such methodology additionally allowed us to determine the relaxation exponent $n$ of the equation for the stiffness of the critical gel. ${ }^{25}$ The value of $n$ varies from 0 (stiff critical gel) to 1 (soft gel); our system presented an intermediate value of 0.53 .

The evolution of complex viscosity and gelling time should be related to the consumption of double bonds from the acrylic functionality. To confirm that the polymerization of the gel was followed with ${ }^{1} \mathrm{H}$ NMR (Figure 5) to assess the decrease in the area of bands associated with acrylic double bonds $(5.7 \mathrm{ppm}$ and $6.0 \mathrm{ppm}$ ). Clearly, the conversion proceeds fast in the first minutes, reaching about $55 \%$ after $15 \mathrm{~min}$ of polymerization. Only about half of the incorporated double bonds, therefore, are required to fully cross-link the polymer if this data is compared to the ones obtained by rheometry. Due to the increase in viscosity and decrease in chain mobility, as a consequence of such widespread cross-linking, conversion increases slowly up to $30 \mathrm{~min}(\sim 62 \%)$ and then levels-off, reaching about $68 \%$ after $1 \mathrm{~h}$ of polymerization. Nonetheless, and although the material is already a hardened gel, polymerization proceeds during the first week, with conversions of up to $76 \%$ at 7 days.

This data means that $24 \%$ of the acrylic double bonds will still remain within the gel even after 1 week of polymerization,

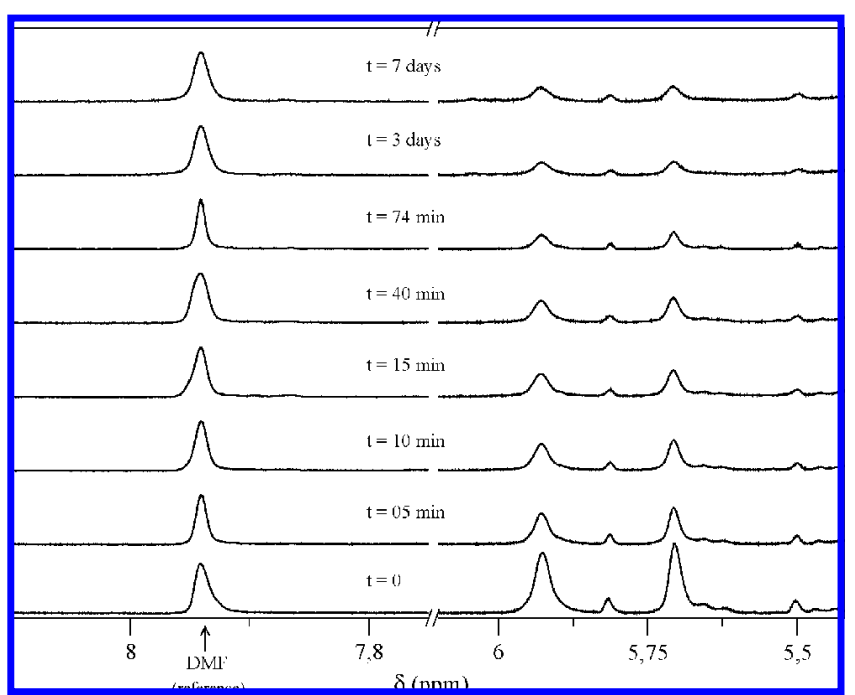

Figure 5. Evolution of the polymerization, expressed as conversion of double bonds, followed by in situ polymerization ${ }^{1} \mathrm{H}$ NMR. The bands at 5.7 and 5.9 ppm corresponds to the vinyl protons; dimethyl formamide (band at $7.95 \mathrm{ppm}$ ) was added as a reference.

which could create potential cytotoxic issues. To evaluate that, the extracts of injectable hydrogels were added to a fibroblast cell line for a period of 1 week. Sterilization was performed by filtration of the oligomer solution, therefore, no subsequent washing or lag time (besides the time for hardening of the gel) was needed. This was an important precaution to ensure that all possibly toxic extractable substance would be assessed. Moreover, methacrylic functionalized oligomers (without crosslinking) were also evaluated to verify the noncytotoxicity of the starting material. After 1 day, the dissolved oligomers showed no significant difference on cellular viability when compared to a Thermanox control, confirming that the acrylic functionality is not cytotoxic. This is probably due to the fact they are attached to relatively large oligomers (MW $700 \mathrm{~g} / \mathrm{mol}$ ), in contrast to the small MMA $(100.12 \mathrm{~g} / \mathrm{mol})$, a toxic acrylate monomer released from self-curing acrylic bone cements. ${ }^{26,27}$ Extractables from the hardened gel (activators, unreacted oligomers, degradable products) were noncytotoxic as well after up to 7 days. In fact, after the second day of testing, the gel showed increased cellular viability as compared to the control, reaching $160 \%$ of the values shown by Thermanox after 7 days (this value was significant with $p<0.05$ ). It seems, therefore, that the released and degraded acrylated oligomers were promoting cell multiplication, an effect already demonstrated for conventional chitooligosaccharides. ${ }^{28}$ These results outperformed prefabricated chitosan membranes, which displayed no significant difference with the control over the entire period.

Last, degradation of injectable materials was assessed in solutions containing lysozyme, an enzyme which degrades chitosan. Degradation solutions were analyzed both by HPLC and by a colorimetric method. ${ }^{16}$ While chitosan membranes showed very slow degradation rate, injectable materials were quickly degraded since the first week. After 9 weeks, injectable materials had lost about $28.1 \%$ of their initial mass, while chitosan membranes, under the same conditions, lost only $0.98 \%$. This is probably due to the much higher water content of the injectable hydrogels, which are composed of $90 \%$ water. It then allows much faster and easier diffusion of the enzyme into the material, speeding up the process of degradation. A large amount of chitooligosaccharides was already detected on the first week (Figure 6), which may explain the higher cellular viability observed for these materials. It is also important to 


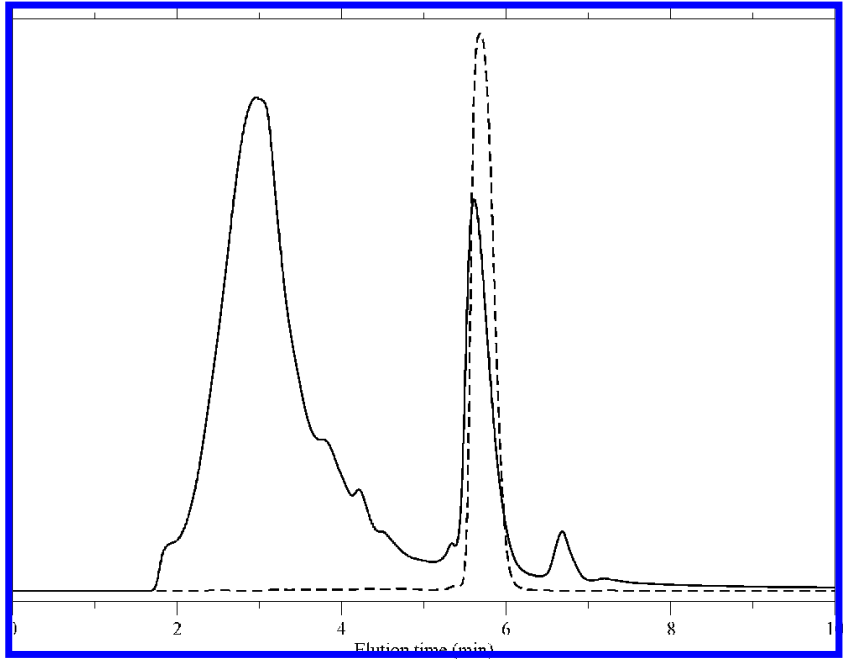

Figure 6. HPLC spectra of degradation products from chitosan membranes after 9 weeks (dashed line) and injectable material after 1 week (solid line). The peak at 5.7 min corresponds to the lysozyme. The degradation products of the chitosan injectable are high MW oligomers (Dp 3; broad peak from $1.8 \mathrm{~min}$ to $4.6 \mathrm{~min}$ ), low MW oligomers (Dp 3; shoulders at $3.8 \mathrm{~min}$ and $4.2 \mathrm{~min}$, peak at $6.9 \mathrm{~min}$ ), and the monomers (D-glucosamine at $3.0 \mathrm{~min}$ and $\mathrm{N}$-acetyl glucosamine at $3.2 \mathrm{~min})$.

stress that, despite such higher weight loss, the specimens did not disintegrate, keeping their initial shape and cohesion even after 80 days of degradation.

\section{Conclusion}

An innovative injectable system composed of methacrylic modified chitooligosaccharides was introduced. The system may be prepared by an easy route and is able to gel in physiological conditions in less than $15 \mathrm{~min}$. The gel presents a shear storage modulus in the $\mathrm{kPa}$ range, therefore, being well suited for drug delivery or soft tissue engineering applications. Degradation and cytotoxicity behavior outperformed chitosan membranes. In summary, injectable biodegradable systems based on chitooligosaccharides are interesting materials to be used in the biomedical field, especially for tissue engineering or drug delivery systems. They perform as well as other injectable or prefabricated materials and are additionally almost entirely composed of biobased polymers. Moreover, their preparation does not make use of toxic solvents or time-consuming intermediate steps. This is in clear contrast with the majority of injectable systems currently being developed and represent an appealing concept for their scale up and industrial production once the production process is simple and may be easily adapted to ensure recovery of byproducts. It may also represent a valuable alternative for less-favored countries to develop their own technology, because the materials and processes described here are cheap and widely available.
Acknowledgment. The authors thank Dr. Mar Fernández for the cytotoxicity tests. L.F.B. thanks the European Commission for supporting this work through a Marie Curie-IIF fellowship.

\section{References and Notes}

(1) Boesel, L. F.; Reis, R. L. In Biodegradable Systems in Tissue Engineering and Regenerative Medicine; Reis, R. L., San Román, J., Eds.; CRC Press: Boca Raton, FL, 2004; Chapter 2, pp 13-28.

(2) Kretlow, J. D.; Klouda, L.; Mikos, A. G. Adv. Drug Deliverv Rev. 2007, 59, 263-273.

(3) Krauland, A. H.; Guggi, D.; Bernkop-Schnürch, A. Int. J. Pharm. 2006, 307, 270-277.

(4) Klouda, L.; Mikos, A. G. Eur. J. Pharm. Biopharm. 2008, 68, 34-45.

(5) Mano, J. F.; Silva, G. A.; Azevedo, H. S.; Malafaya, P. B.; Sousa, R. A.; Silva, S. S.; Boesel, L. F.; Oliveira, J. M.; Santos, T. C.; Marques, A. P.; Neves, N. M.; Reis, R. L. J. R. Soc. Interface 2007, 4, 999-1030.

(6) Jeong, B.; Windisch Jr, C. F.; Park, M. J.; Sohn, Y. S.; Gutowska, A.; Char, K. J. Phys. Chem. B 2003, 107, 10032-10039.

(7) Lee, J.; Bae, Y.; Sohn, Y.; Jeong, B. Biomacromolecules 2006, 7, $1729-1734$

(8) Fisher, J. P.; Timmer, M. D.; Holland, T. A.; Dean, D.; Engel, P. S.; Mikos, A. G. Biomacromolecules 2003, 4, 1327-1334.

(9) Wang, S.; Kempen, D. H.; Simha, N. K.; Lewis, J. L.; Windebank, A. J.; Yaszemski, M. J.; Lu, L. Biomacromolecules 2008, 9, 12291241.

(10) Anseth, K. S.; Quick, D. J. Macromol. Rapid Commun. 2001, 22, 564 572.

(11) Shastri, V. P.; Padera, R. F.; Tarcha, P.; Langer, R. Biomaterials 2004, $25,715-721$.

(12) Ohya, S.; Matsuda, T. J. Biomater. Sci., Polym. Ed. 2005, 16, 809827.

(13) Cho, J. H.; Kim, S.-H.; Park, K. D.; Jung, M. C.; Yang, W. I.; Han, S. W.; Noh, J. Y.; Lee, J. W. Biomaterials 2004, 25, 5743-5751.

(14) Chung, H. J.; Go, D. H.; Bae, J. W.; Jung, I. K.; Lee, J. W.; Park, K. D. Curr. Appl. Phvs. 2005, 5, 485-488.

(15) Lavertu, M.; Xia, Z.; Serreqi, A. N.; Berrada, M.; Rodrigues, A.; Wang, D.; Buschmann, M. D.; Gupta, A. J. Pharm. Biomed. Anal. 2003, 32, $1149-1158$

(16) Dubois, M. Anal. Chem. 1956, 28, 350-356.

(17) Cabrera, J. C.; Messiaen, J.; Cambier, P.; Van Cutsem, P. Phvsiol. Plant. 2006, 127, 44-56.

(18) Cabrera, J. C.; Van Cutsem, P. Biochem. Eng. J. 2005, 25, 165-172.

(19) Temenoff, J. S.; Shin, H.; Conway, D. E.; Engel, P. S.; Mikos, A. G. Biomacromolecules 2003, 4, 1605-1613.

(20) Williams, C. G.; Malik, A. N.; Kim, T. K.; Manson, P. N.; Elisseeff, J. H. Biomaterials 2005, 26, 1211-1218.

(21) Najjar, A. M. K.; Yunus, W. M. Z. W.; Ahmad, M. B.; Rahman, M. Z. A. J. Appl. Polvm. Sci. 2000, 77, 2314-2318.

(22) Beniash, E.; Hartgerink, J. D.; Storrie, H.; Stendahl, J. C.; Stupp, S. I. Acta Biomater. 2005, 1, 387-397.

(23) Dang, J. M.; Sun, D. D. N.; Shin-Ya, Y.; Sieber, A. N.; Kostuik, J. P.; Leong, K. W. Biomaterials 2006, 27, 406-418.

(24) Krauland, A. H.; Hoffer, M. H.; Bernkop-Schnürch, A. Drug Dev. Ind. Pharm. 2005, 31, 885-893.

(25) Winter, H. H.; Mours, M. Adv. Polvm. Sci. 1997, 134, 165-234.

(26) Yoshii, E. J. Biomed. Mater. Res. 1997, 37, 517-524.

(27) Taira, M.; Nakao, H.; Matsumoto, T.; Takahashi, J. Int. J. Prosthodont. 2000, 13, 311-315.

(28) Minami, S.; Okamoto, Y.; Shigemasa, Y.; Saimoto, H.; Morimoto, M.; Okamura, Y.; Tsuka, T.; Renbutsu, E.; Kubomura, D.; Matahira, Y.; Sakamoto, K.; Omura, Y. In Advances in Chitin Science; Şenel, S., Vårum, K. M., Şumnu, M. M., Hincal, A. A., Eds.; Alp Ofset: Ankara, Turkey, 2007; pp 199-206.

BM801332U 